\title{
Mosquitos de ambientes peri e extradomiciliares na região sul do Brasil ${ }^{\star}$
}

\section{Mosquitoes of peridomiciliary and extradomiciliary environments in of southern Brazil}

\author{
Uelsei Teodoro**, Ana Lúcia Falavigna Guilherme**, Ana Leuch Lozovei***, Vicente La Salvia

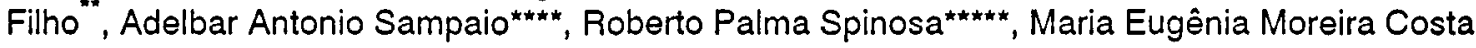 \\ Ferreira ${ }^{\star \star \star \star \star \star}$, Orlando Carlos Barbosa ${ }^{\star \star \star \star \star}$, Edson Maurício de Lima ${ }^{\star \star}$
}

\begin{abstract}
TEODORO, U. et al. Mosquitos de ambientes peri e extradomiciliares na regiño sul do Brasil. Rev. Saúde Pública, 28: 107-15, 1994. Dumnte um ano de colctas de culicideos no Municipio de Querência do Norte, no Estado do Paraná, Brasil, utilizando-se isca humana e armadilhas de Falcão, investigou-se a composição faunística, a sazonalidade, o horurio de maior densidade, a afinidade ao hospedeiro humano $\mathrm{c}$ a presença desses dípteros $\mathrm{cm}$ abrigos de animais domésticos. De junho de 1989 a maio de 1990 foram coletados 5.923 mosquitos dos gêneros Aedes, Aedomyia, Coquillettidia, Culex, Mansonia, Psorophora, Sabethes e Uranotaenia. Identificaram-se 32 espécies de culicideos, dentre as quais Aedes scapularis, Anopheles albitarsis, Acdomyia squamipennis, Coquillettialia lynchi, Mansonia tillllans e Coquillellidia venezuelensis tiveram maior prevalência, tendo sido capturados em grande número $\mathrm{cm}$ isca humana, cxceto Aedomyia squamipennis que compareceu sobretudo cm abrigos de animais domésticos. O horário de maior atividade foi entre 18 e 19 horas e o mês de maior densidade foi o de abril, considerando-se o conjunto dos insetos capturados.
\end{abstract}

Descritores: Mosquitos. Ecologia de vetores.

\section{Introdução}

O papel dos culicídeos como vetores de parasitos humanos c de animais clomésticos tem despertado o interesse de se conhecer a fauna e o comportamento desses insetos nos diversos ambientes, sobretudo naqueles modificados e freqüentados pelo homem c animais domésti$\cos$.

Ressalta-se que Anopheles albitarsis e Anopheles darlingi têm most rado clevadas densidades no Estado do Parana ${ }^{3,4}$, cabendo lcmbrar que essas espécies são vctoras dos plasmó-

\footnotetext{
* Financiado pelo Consclho de Ciência e Tecnologia do Estado do Paraná (CONCIITC).

* Departamento de Análises Clinicas da Universidade Estadual de Maringá - Maringá, PR - Brasil

*** Departamento de Patologia da Universidade Federal do Paraná - Centro Politécnico - Curitiba, PR - Brasil

**** Departamento de Estatística da Universidade Istadual de Maringá - Maringá, PR - Brasil

***** Fundação Nacional de Saúde do Ministério da Saúde - Londriun, PR - Brasil

****** Departamento de Geografia da Universidade Estadual de Maringá - Maringá, PR - Brasil

Separatas/Reprints: U. Tcodoro - Av. Colombo, $3690-87020$ -900 - Maringá, PR - Brasil
}

dios da malária humana ${ }^{5}$, c que no citado Estado é fireqüente a migração de e para áreas endêmicas, no Norte do Brasil ${ }^{1,19,20}$.

$\Lambda$ freqüência elevada de Aedes scapularis em isca humana e em ambientes degradados tcm confirmado a antropolilia e a possivel adaptação desse inseto nos ambientes alterados $9,10,15$, destaciando-se também que esse mosquito provavelmente esteja associado ao vírus da cncefalite ${ }^{15}$ e outros arbovírus.

O presente trabalho teve como objetivos a investigação sobre a composição da fauna de culicídcos, o horário de maior atividade, a sazonalidade, a afinidacle em relação ao hospedeiro humano $\mathrm{c}$ a presença desses dípteros cm abrigos de animais domésticos numa área em que o ambicnte foi profundamente altcrado e hoje predomina a pecuária.

\section{Material e Método}

As capturas de culicídcos foram efetuadas na fazenda Sonho Real, propriedade da Jabur Agropecuária Ltcla., no Município de Querência do Norte, no norocste do Estado do Paraná (Figura). 


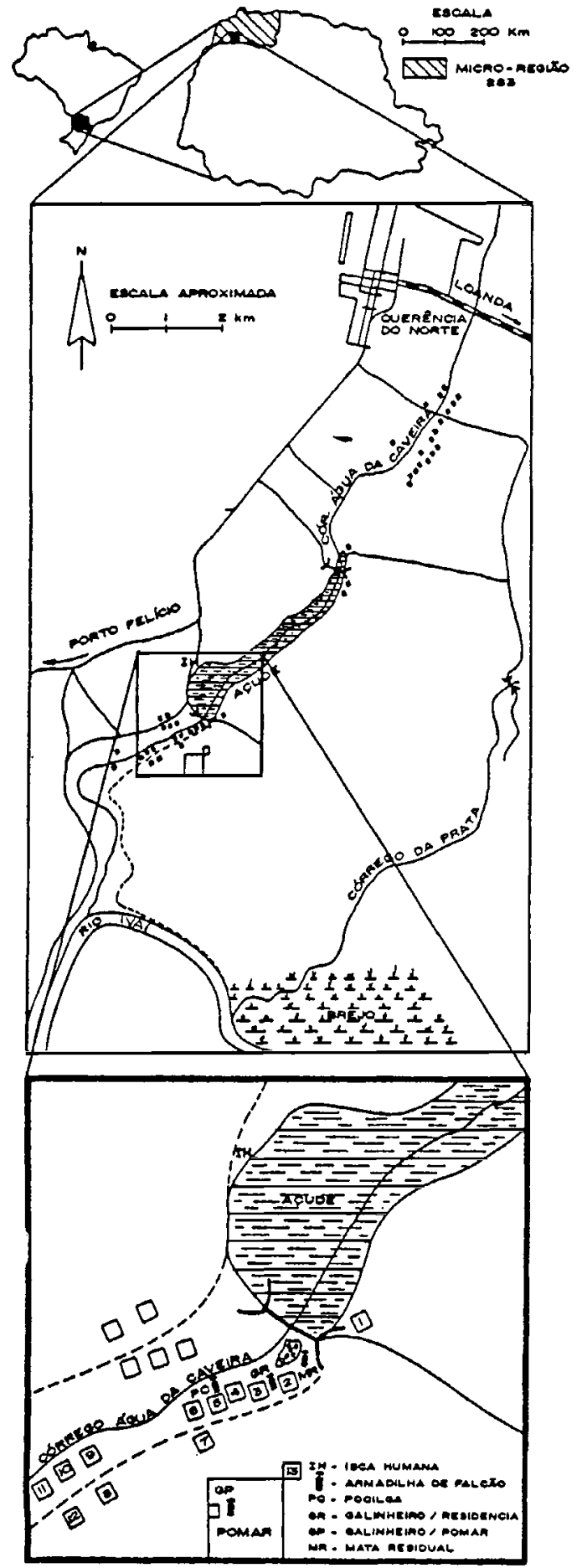

Figura 1 - Locais de capturas de culicídeos na fazenda Sonho Real, Municipio de Querência do Norte, Estado do Paraná.
Os aspectos geográficos gerais da região noroeste do Estado do Paraná correspondem à descrição da Fundação Instituto Brasileiro de Gcografia e Estatística ${ }^{11}$ (1990), de Maack ${ }^{18}$ (1968) e da Universidade Estadual de Maringá ${ }^{22}$ (1991), conforme segue abaixo:

1. o clima regional é Tropical Subquente úmido, com um a dois meses de seca no inverno (julho e agosto), pluviosidade de 1.250 a 1.500 $\mathrm{mm} / \mathrm{ano}$, verão quente (média de $24-26^{\circ} \mathrm{C} \mathrm{em}$ janciro e máxima de $40^{\circ} \mathrm{C}$ ), com eventuais geadas (cinco a dez dias/ano);

2. quanto à gcologia, a região insere-se no domínio da bacia do Paraná, na porção afetada pelo derrame de lavas básicas recobertas por formações areníticas;

3. com relação ao relevo, a região corresponde a uma parcela do Terceiro Planalto Paranaense; o rio Ivaí apresenta no trecho final (onde se insere a área das capturas de culicídeos) curso sinuoso com nítidos processos de acumulaçĩo de sedimentos em terraços e em planícies aluviais de pequeno porte; banhados ou áreas brejosas estendem-se até 4 ou $5 \mathrm{~km}$ a partir das margens;

4. o solo é arenoso à superlicie, com incremento variável de argila $\mathrm{em}$ profundidade, e costuma ser suscetível à crosão e voçorocamento. Como conseqüência tem-se o assoreamento inferior do rio Ivaí e a formação de planícies de inundação cortadas por canais anastomosados com muitas lagoas rasas, idcais para a proliferação de mosquitos. Por ser de fertilidade relativamente baixa, o solo é utilizado para pastagens e cultivos temporários de mandioca, algodão, milho, cana-de-açúcar e mais raramente trigo, soja ou café;

5. a vegetação primitiva de mata - Floresta Estacional Semidecidual - , que recobria extensivamente a região até a década de 60 , desapareceu quase totalmente em face da ocupação agrária. Raros capões de mata isolados, nos amplos interflúvios, e matas ciliares descontínuas acompanham os cursos d'água. São manchas de mata secundária, apresentando uma vegetação mais baixa mas bastante intrincada;

6. a área onde foram efetuadas as coletas, na fazenda Sonho Real, apresenta edificações rústicas construídas ao longo do córrego Água da Caveira, um afluente da margem direita do rio Ivaí, com extensão total de cerca de $30 \mathrm{~km}$. 
Tabela 1. Culicideos coletados com isca humana em horários matutino e vespertino, às margens de um açude formado pelas águas do córrego Água da Caveira, na fazenda Sonho Real, Município de Queréncia do Norte, Estado do Paraná, Brasil, de junho de 1989 a maio de 1990.

\begin{tabular}{|c|c|c|c|c|c|c|c|c|c|c|}
\hline \multirow[t]{2}{*}{ Espécie } & \multicolumn{8}{|c|}{ Horário } & \multirow[t]{2}{*}{ Total } & \multirow[t]{2}{*}{$\%$} \\
\hline & $4-5$ & 5.6 & $6-7$ & 7.8 & $16-17$ & $17 \cdot 18$ & 18.19 & $19-20$ & & \\
\hline An. Albitarsis & 25 & 45 & 30 & 1 & 1 & 83 & 350 & 144 & 649 & 15,23 \\
\hline An. darlingi & $\cdot$ & 1 & 1 & - & - & - & - & - & 2 & 0,05 \\
\hline An. evansae & 10 & 4 & 23 & 3 & - & - & 75 & 43 & 158 & 3,71 \\
\hline An. galvaol & - & 1 & 11 & - & - & 1 & 21 & 9 & 43 & 1,01 \\
\hline An./utzl & 3 & - & - & - & - & - & 1 & - & 4 & 0,09 \\
\hline An. rondoni & 4 & 4 & 24 & - & - & 8 & 38 & 15 & 93 & 2,18 \\
\hline An.triannulatus & 1 & - & 6 & - & - & 13 & 23 & 16 & 59 & 1,38 \\
\hline Anopheles spp. & 2 & 2 & 6 & 1 & - & 3 & 33 & 24 & 71 & 1,66 \\
\hline Ae. scapularis & 36 & 144 & 413 & 24 & 1 & 181 & 437 & 193 & 1.429 & 33,53 \\
\hline A日. serratus & - & 1 & - & - & - & - & 2 & 3 & 6 & 0,14 \\
\hline Aedes sp. & - & 8 & - & - & - & - & 4 & 6 & 18 & 0,42 \\
\hline Ad. squamipennis, & - & - & - & - & - & - & - & 1 & 1 & 0,02 \\
\hline Cq. juxtamansonia & 2 & - & - & - & - & - & - & - & 2 & 0,05 \\
\hline Cq. Iynchi & 51 & 53 & 44 & 1 & - & - & 5 & 169 & 323 & 7,58 \\
\hline Cq. shannoni & 2 & - & 2 & - & - & - & - & 8 & 12 & 0,28 \\
\hline Cq. venezuelensis & 26 & 6 & 111 & - & - & - & 8 & 26 & 177 & 4,15 \\
\hline Cx. acharistus & - & - & - & - & - & - & 7 & - & 7 & 0,16 \\
\hline Cx. bonneae & - & - & - & - & - & - & 1 & 3 & 4 & 0,09 \\
\hline Cx. chidesteri & 1 & 2 & - & - & - & - & 13 & - & 16 & 0,38 \\
\hline Cx. coronator & 3 & - & - & - & - & - & 5 & 11 & 19 & 0,45 \\
\hline Cx. declarator & 4 & - & - & - & - & - & 8 & 3 & 15 & 0,35 \\
\hline Cx. mollis & - & - & - & - & - & - & 22 & 3 & 25 & 0,59 \\
\hline Cx.quinquefasciatus & - & 3 & - & - & - & - & 23 & 5 & 31 & 0,73 \\
\hline Cx. (Culex) spp. & - & - & 9 & - & - & - & 16 & 17 & 42 & 0,99 \\
\hline Cx. (Melanoconion) spp. & - & 15 & 26 & - & - & - & 22 & 99 & 162 & 3,80 \\
\hline Culex spp. & 2 & - & - & - & - & - & - & 26 & 28 & 0,66 \\
\hline Ma. titillans & 7 & 10 & 13 & - & 1 & 1 & 85 & 107 & 224 & 5,25 \\
\hline Ma. wilsoni & - & - & - & - & - & - & - & 3 & 3 & 0,07 \\
\hline Mansonia sp. & - & 2 & - & - & - & - & - & 1 & 3 & 0,07 \\
\hline Ps. ciliata & - & - & - & 1 & - & 1 & 2 & 3 & 7 & 0,16 \\
\hline Ps. confinnis & 1 & 1 & 4 & - & 1 & 2 & 24 & 36 & 69 & 1,62 \\
\hline Ps. discolor & 1 & - & - & - & - & - & - & - & 1 & 0,02 \\
\hline Ps. ferox & - & - & - & - & - & - & - & 1 & 1 & 0,02 \\
\hline Ps. lanei & - & 2 & - & - & - & - & - & - & 2 & 0,05 \\
\hline Ps. varipes & 5 & 28 & 77 & 13 & 7 & 36 & 159 & 98 & 423 & 9,93 \\
\hline Ps. (Janthinosoma) sp. & - & - & - & - & 1 & - & - & 8 & 9 & 0,21 \\
\hline Psorophora spp. & - & 59 & - & 1 & - & - & 1 & 63 & 124 & 2,90 \\
\hline Sa. (Sabethoides) sp. & - & - & - & - & - & 1 & - & - & 1 & 0,02 \\
\hline Total & 186 & 361 & 800 & 45 & 12 & 330 & 1.386 & 1.143 & 4.263 & \\
\hline$\%$ & 4,36 & 8,47 & 18,77 & 1,06 & 0,28 & 7,74 & 32,51 & 26,81 & 100,00 & \\
\hline
\end{tabular}


O local das capturas situa-se na porção final do córrego, distando de 7 a $8 \mathrm{~km}$ da sua confluência com o rio Ivaí, a uma altitude em torno de 230-240m, nos terrenos planos de antigas várzeas e de terraços aluviais. A montante do núcleo residencial o córrego Água da Caveira foi barrado, formando um pequeno açude. Margeando o curso d'água, imediatamente atrás das casas, há uma estreita faixa de mata ciliar (Figura).

As capturas ocorreram de junho de 1989 a maio de 1990, tanto com isca humana quanto com as armadilhas de Falcão ${ }^{6}$ (1981). As coletas foram mensais, em dois dias consecutivos em isca humana, e apenas um dia com as armadilhas de Falcão.

As coletas com isca humana eram feitas por dois indivíduos, das 4 às $8 \mathrm{~h}$ e das 16 às $20 \mathrm{~h}$, num total de 192 horas, às margens do açude formado pelas águas do córrego Água da Caveira. As armadilhas de Falcão eram instaladas das 21 às $3 \mathrm{~h}$, perfazendo 72 horas de coletas cada armadilha. Estas armadilhas foram distribuídas da seguinte forma: uma na mata residual (MR), abaixo do açude; uma dentro de um galinheiro (GR) entre as residências 2 e 3; outra dentro de um galinheiro (GP) no interior de um pomar; e a última dentro de uma pocilga (PC), nos fundos da residência 5 (Figura).

A análise estatística dos resultados foi feita utilizando-se o teste do qui-quadrado e o teste da diferença de duas proporções, a nível de $5 \%$ de significância ( $\mathrm{p}<0,05)$, do "software" estatístico SYSTAT.

\section{Resultados}

Em isca humana houve diferença entre o número de mosquitos capturados nos períodos das 4 às $8 \mathrm{~h}$ e das 16 às $20 \mathrm{~h}$, e entre as horas que compõem esses períodos $\left(x^{2}=3.514,08\right)$; o horário de maior prevalência dos culicídeos foi das 18 às $19 \mathrm{~h}(32,51 \%)$, seguindo-se os horários das 19 às $20 \mathrm{~h}(26,81 \%)$ e das 6 às 7 h $(18,77 \%)$ (Tabela 1).

No conjunto dos mosquitos capturados em isca humana ocorreu diferença entre as espécies prevalentes $\left(\mathrm{x}^{2}=1.534,70\right) ;$ Ae. scapularis teve maior freqüência $(33,53 \%)$, vindo em seguida $A n$. albitarsis $(15,23 \%)$, Ps. varipes
(9,93\%), Cq. lynchi $(7,58 \%)$ e Ma. titillans $(5,25 \%)$ (Tabela 1 ).

Ae. scapularis compareceu em maior número das 18 às $19 \mathrm{~h}(30,58 \%)$, seguindo-se os horários das 6 às $7 \mathrm{~h}(28,90 \%)$ e das 19 às $20 \mathrm{~h}$ (13,51\%) ( $\left.\mathrm{x}^{2}=796,61\right) ; A n$. albitarsis foi mais freqüente das 18 às $19 \mathrm{~h}(53,92 \%)$, e, em seguida, entre 19 e $20 \mathrm{~h}(22,19 \%)\left(\mathrm{x}^{2}=756,75\right) ;$ Ps. varipes teve maior presença entre 18 e $19 \mathrm{~h}$ $(37,59 \%)$, das 19 às $20 \mathrm{~h}(23,17 \%)$ e das 6 às $7 \mathrm{~h}$ $(18,20 \%)\left(x^{2}=194,57\right)$; Cq. lynchi foi mais freqüente entre 19 e $20 \mathrm{~h}(52,53 \%)$ e das 4 às $6 \mathrm{~h}$ $(32,20 \%)\left(x^{2}=128,65\right)$; e Ma. titillans prevaleceu das 19 às $20 \mathrm{~h}(47,77 \%)$ e entre 18 e $19 \mathrm{~h}$ $(37,95 \%)\left(x^{2}=159,50\right)$ (Tabela 1$)$.

Nas armadilhas de Falcão houve diferença no número de mosquitos capturados nos vários ecótopos ( $\left.x^{2}=53,67\right)$; a pocilga (PC) foi o ecótopo onde ocorreu o maior número de mosquitos (30,06\%), e a seguir os ccótopos GP (27,59\%), MR $(24,28 \%)$ e GR $(18,07 \%)$ (Tabela 2$)$.

Ocorreu diferença entre os culicídeos de maior prevalência no conjunto dos ecótopos $\left(\mathrm{x}^{2}=1.534,72\right)$; o mosquito mais freqüente foi Ad. squamipennis $(25,55 \%)$; seguido por espé. cies de Cx. (Melanoconion) (14,77\%), An. albitarsis $(8,43 \%), C x$. quinquefasciatus $(7,41 \%)$; Ae. scapularis $(6,45 \%)$, Ma. titillans $(5,42 \%)$, e em sétimo lugar $(z=0,39) C x$. (Culex) spp. $(5,56 \%)$ e Cx. coronator $(5,06 \%)$ (Tabela 2$)$.

No ecótopo PC Ad. squamipennis $(22,24 \%)$ prevaleceu sobre $A n$. albitarsis (21,24\%), Ma. titillans $(8,82 \%)$, An. rondoni (7,01\%), Cx. (Melanoconion) spp. (6,41\%) Ae. scapularis $(6,21 \%)\left(x^{2}=175,61\right)$; em GP Ad. squamipennis $(33,41 \%)$ teve maior freqüência que Ae. scapularis $(12,88 \%)$, espécies de $C x$. (Culex) $(11,80 \%)$ e espécies de $C x$. (Melanoconion) $(10,30 \%)\left(x^{2}=281,89\right)$; no ecótopo MR prevaleceram espécies de $C x$. (Melanoconion) (26,30\%), seguindo-se Ad. squamipennis $(21,84 \%)$, Cx. quinquefasciatus $(10,92 \%), \mathrm{Cu}$ lex spp. $(10,17 \%)$ e C $x$. coronator $(8,44 \%)$ $\left(\mathrm{x}^{2}=119,18\right)$; em GR observou-se a prevalência de Ad. squamipennis $(24,0 \%) \mathrm{e}$, na seqüência, espécies de $C x$. (Melanoconion) (20,0\%), $C x$. quinquefasciatuss $(15,67 \%)$ e C $x$. (Culex) spp. $(10,0 \%)\left(x^{2}=94,50 \%\right)$ (Tabela 2$)$.

Ocorreu diferença na quantidade de culicídeos capturados nos meses de junho de 1989 a maio de $1990\left(x^{2}=2.147,54\right)$; o mês de maior 
Tabela 2. Culicídeos coletados com armadilhas de Falcão em abrigos de animais domésticos, na fazenda Sonho Real, Municipio de Querencia do Norte, Estado do Paraná, Brasil, de junho de 1989 a maio de 1990.

\begin{tabular}{|c|c|c|c|c|c|c|}
\hline \multirow[t]{2}{*}{ Espécie } & \multicolumn{4}{|c|}{ Armadilha } & \multirow[t]{2}{*}{ Total } & \multirow[t]{2}{*}{$\%$} \\
\hline & $M R$ & GR & GP & $\mathrm{PC}$ & & \\
\hline An. albitarsis & - & 15 & 19 & 106 & 140 & 8,43 \\
\hline An. argyritarsis & - & - & - & 6 & 6 & 0,36 \\
\hline An. evansae & 2 & 1 & 5 & 20 & 28 & 1,69 \\
\hline An. galvaoi & 4 & - & - & - & 4 & 0,24 \\
\hline An. mattogrossenais & - & - & - & 2 & 2 & 0,12 \\
\hline An. rondoni & 2 & 4 & - & 35 & 41 & 2,47 \\
\hline An. triannulatus & 6 & 4 & - & 15 & 25 & 1,51 \\
\hline An. (Nyssorhynchus) sp. & 1 & - & - & 10 & 11 & 0,66 \\
\hline Anopheles spp. & 4 & - & 1 & 10 & 15 & 0,90 \\
\hline Ae. scapularis & 6 & 11 & 59 & 31 & 107 & 6,45 \\
\hline Ae. serratus & - & 1 & - & - & 1 & 0,06 \\
\hline Ad. squamipennis & 88 & 72 & 153 & 111 & 424 & 25,55 \\
\hline Cq. Iynchi & - & - & 1 & 5 & 6 & 0,36 \\
\hline Cq. shannoni & - & - & - & 2 & 2 & 0,12 \\
\hline Cq. venezuelensis & - & 6 & 9 & 5 & 15 & 1,20 \\
\hline Cx. amazonensis & 2 & - & - & - & 2 & 0,12 \\
\hline Cx. chidesteri & 1 & - & 1 & - & 2 & 0,12 \\
\hline Cx. coronator & 34 & 18 & 29 & 3 & 84 & 5,06 \\
\hline Cx. declarator, & 6 & 4 & 12 & - & 22 & 1,33 \\
\hline Cx. mollis & - & - & 2 & - & 2 & 0,12 \\
\hline Cx. quinquefasciatus & 44 & 47 & 20 & 12 & 123 & 7,41 \\
\hline Cx (Culex) spp. & 3 & 30 & 54 & 2 & 89 & 5,36 \\
\hline Cx. (Melanoconion) spp. & 106 & 60 & 47 & 32 & 245 & 14,77 \\
\hline Culex spp. & 41 & 8 & 24 & 26 & 99 & 5,96 \\
\hline Ma. titillans & 24 & 4 & 18 & 44 & 90 & 5,42 \\
\hline Mansonia sp. & - & 3 & - & - & 3 & 0,18 \\
\hline Ps. cillata & - & - & 1 & - & 1 & 0,06 \\
\hline Ps. confinnis & 1 & 1 & - & 5 & 7 & 0,42 \\
\hline Sabethes sp. & 2 & - & - & - & 2 & 0,12 \\
\hline Uranotaenia sp. & 26 & 11 & 3 & 17 & 57 & 3,43 \\
\hline Total & 403 & 300 & 458 & 499 & 1.660 & \\
\hline$\%$ & 24,28 & 18,07 & 27,59 & 30,06 & & 100,00 \\
\hline
\end{tabular}

$M R=$ mata residual alterada, abaixo do açude

$\mathrm{GR}=$ galinheiro entre as residèncias 2 e 3

$\mathrm{GP}=$ galinheiro situado na margem do pomar 
Tabela 3. Variação estacional de culicideos coletados com isca humana às margens de um açude formado pelas àguas do córrego Água da Caveira, na fazenda Sonho Real, Município de Queréncia do Norte, Estado do Paraná, Brasil, de junho de 1989 a majo de 1990.

\begin{tabular}{|c|c|c|c|c|c|c|c|c|c|c|c|c|c|}
\hline \multirow{2}{*}{ Espécie } & \multicolumn{7}{|c|}{1989} & \multicolumn{5}{|c|}{1990} & \multirow[t]{2}{*}{ Total } \\
\hline & Jun & Jul & Ago & Set & Out & Nov & Dez & Jan & Fev & Mar & Abr & Mai & \\
\hline An. albitarsis & 66 & 162 & 44 & 30 & 40 & 18 & 9 & - & 49 & 11 & 101 & 127 & 649 \\
\hline An. darlingi & - & - & - & - & - & - & - & - & 2 & - & - & - & 2 \\
\hline An. evansae & 49 & 25 & 6 & - & 37 & 24 & - & - & 2 & - & 6 & 9 & 158 \\
\hline An. galvaoi & - & - & 9 & 7 & - & - & - & - & 1 & - & 10 & 16 & 43 \\
\hline An. rondoni & 29 & 13 & 1 & - & 3 & 5 & - & - & - & 3 & 31 & 8 & 93 \\
\hline An. triannulatus & 11 & 4 & - & 2 & - & - & - & - & - & 3 & 22 & 17 & 59 \\
\hline An. lutzi & - & - & - & - & - & - & - & - & - & - & 3 & 1 & 4 \\
\hline Anopheles spp. & 9 & - & - & 34 & 6 & - & - & - & 2 & - & 18 & 2 & 71 \\
\hline Ae. scapularis & 76 & 103 & 109 & 32 & 21 & 33 & 72 & 177 & 41 & 12 & 676 & 77 & 1.429 \\
\hline Ae. serratus & - & - & - & - & 2 & - & - & - & - & - & 4 & - & 6 \\
\hline Aөdes spp. & - & - & 1 & - & - & - & - & 10 & 7 & - & - & - & 18 \\
\hline Ad. squamipennis & - & - & - & 1 & - & - & - & - & - & - & - & - & 1 \\
\hline Cg. juxtamansonia & - & - & - & - & - & - & - & 2 & - & - & - & - & 2 \\
\hline Cq. Iyncyhi & - & - & - & - & 4 & - & - & - & 262 & 31 & 25 & 1 & 323 \\
\hline Cq. shannoni & - & - & - & - & - & - & - & - & 10 & 2 & - & - & 12 \\
\hline Cq, venezuelensis & 10 & - & - & - & 19 & 125 & 3 & - & - & - & 20 & - & 177 \\
\hline Cx. bonneae & - & - & 1 & - & - & - & - & - & - & - & 3 & - & 4 \\
\hline Cx. acharistus & - & 5 & - & - & - & - & - & - & - & - & - & 2 & 7 \\
\hline Cx. chidesteri & - & 3 & - & 12 & - & - & - & - & - & - & 1 & - & 16 \\
\hline Cx. coronator & - & - & 2 & - & 8 & 3 & - & - & 2 & - & 1 & - & 16 \\
\hline Cx. declarator & 3 & - & 9 & - & - & - & - & - & - & - & 4 & 2 & 18 \\
\hline Cx. mollis & 22 & 3 & - & - & - & - & - & - & - & - & - & - & 25 \\
\hline Cx. quinquefasciatus & 12 & 5 & 4 & - & - & - & - & - & 1 & 1 & 8 & - & 31 \\
\hline Cx. (Culex) spp. & - & - & - & 15 & 8 & 9 & 9 & - & - & - & 1 & - & 42 \\
\hline Cx. (Melanoconion) spp. & - & - & 10 & 68 & 33 & 23 & 5 & 3 & 16 & - & 4 & - & 162 \\
\hline Culex spp. & - & - & - & 26 & - & 1 & - & 1 & - & - & - & - & 28 \\
\hline Ma. titillans & 19 & 13 & 34 & 77 & 37 & 14 & 7 & - & 8 & 7 & 8 & - & 224 \\
\hline Ma. wilsoni & - & - & - & - & 2 & - & - & - & 1 & - & - & - & 3 \\
\hline Mansonia sp. & - & - & - & 1 & - & - & - & 2 & - & - & - & - & 3 \\
\hline Ps. ciliata & - & - & 1 & 4 & - & - & - & 1 & - & - & 1 & - & 7 \\
\hline Ps. confinnis & 1 & - & 1 & 22 & - & - & - & - & - & - & 45 & - & 69 \\
\hline Ps. discolor & - & - & - & 1 & - & - & - & - & - & - & - & - & 1 \\
\hline Ps. ferox & - & - & - & 1 & - & - & - & - & - & - & - & - & 1 \\
\hline Ps. lanei & - & - & - & - & - & - & - & 2 & - & - & - & - & 2 \\
\hline Ps. varipes & - & - & 5 & 235 & - & 2 & 1 & 69 & 33 & 2 & 76 & - & 423 \\
\hline Ps. (Janthinosoma) sp. & - & - & - & - & - & - & - & 6 & - & - & - & - & 6 \\
\hline Psorophora spp. & - & - & 1 & 2 & - & - & - & 121 & 3 & - & - & - & 127 \\
\hline Sa. (Sabethoides) sp. & 1 & - & - & - & - & - & - & - & - & - & - & - & 1 \\
\hline Total & 308 & 336 & 238 & 571 & 221 & 257 & 98 & 394 & 440 & 72 & 1.068 & 262 & 4.263 \\
\hline
\end{tabular}


densidade foi abril $(25,05 \%)$, seguido dos meses de setembro $(13,39 \%)$ e fevereiro $(10,32 \%)$, ocorrendo em março a menor densidade (1,69\%) (Tabela 3).

Em abril Ae. scapularis teve maior presença (63,30\%), vindo em seguida $A n$. albitarsis (9,50\%) e Ps. varipes $(7,52 \%)\left(\mathrm{x}^{2}=1.197,14\right)$; em setembro os mosquitos com maior densidade foram Ps. varipes (41,16\%), Ma. titillans (13,50\%) e espécies de $C x$. (Melanoconion) $(11,90 \%)\left(x^{2}=309,46\right)$; em fevereiro Cq. lynchi teve maior densidade $(58,50 \%)$ que $A n$. albitarsis $(11,14 \%)$ e Ae. scapularis (9,30\%) $\left(\mathrm{x}^{2}=375,11\right)$ (Tabela 3).

Ae. scapularis foi que teve maior freqüência em todo o período de capturas, com destaque para o mês de abril, quando ocorreu a maior densidade $(47,30 \%)$, seguindo-se janeiro $(12,40 \%)$ e agosto $(7,60 \%)\left(x^{2}=2.309,30\right)$; a segunda espécie com maior densidade foi $A n$. albitarsis que prevaleceu em julho $(25,0 \%)$, depois em maio $(19,60 \%)$ e abril $(15,60 \%)$ $\left(x^{2}=257,32\right) ; P$ s. varipes, a terceira espécie em prevalência compareceu sobretudo em setembro $(55,6 \%)$ e, em seguida, em abril $(17,96 \%)$ e janeiro $(16,31 \%)\left(x^{2}=236,35\right)$; o quarto mosquito mais freqüente foi $C q$. lynchi, que teve maior densidade em fevereiro $(81,11 \%)$, depois em março $(9,59 \%)$ e abril $(7,74 \%)\left(x^{2}=351,60\right) ; a$ quinta espécie prevalente foi Ma. titillans que teve maior presença em setembro $(34,37 \%) \mathrm{e}$, a seguir em outubro $(16,51 \%)$ e agosto $(15,18 \%)$ $\left(x^{2}=887,06\right)($ Tabela 3$)$.

\section{Discussão}

Os mosquitos aqui identificados já foram assinalados no Estado do Paraná por vários autores $2,3,4,7,12,13,14,21$, os quais mostraram que há prevalência de um pequeno número de espécies de mosquitos, para cada localidade investigada. Além disso as espécies prevalentes são distintas para cada uma das localidades.

Numa área do Município de Terra Boa, no Estado do Paraná, e a mais próxima de Querência do Norte (perto de $180 \mathrm{~km}$ ), investigada por Barbosa e col. ${ }^{2}$ (1993), através de coletas com armadilhas de Shannon e Falcão, verificaram-se 30 espécies de culicídeos, com apenas 6 delas Ch.fajardoi, Ae. scapularis, Cx. quinquefas- ciatus, Cq. venezuelesis, $C x$. coronator $e C x$. mollis - representando $61,1 \%$ dos insetos coletados.

Na fazenda Sonho Real, onde foi feito o presente trabalho e a modificação ambiental é mais acentuada, foram constatadas 32 espécies de mosquitos, dos quais sete - Ae. scapularis, An. albitarsis, Ad. squamipennis, Ps. varipes, Cq. lynchi, Ma. titillans e Cq. venezuelensis perfizeram $67,62 \%(4.013 / 5.923)$ do conjunto de mosquitos coletados em isca humana e armadilhas de Falcão. Ae. scapularis e Cq.venezuelensis estão entre os mosquitos de maior prevalência nos dois municípios citados. Ae. scapularis foi significativamente mais freqüente nas capturas em isca humana. Isso confirma as observações de outros autores $8,9,15,16$ sobre a possível adaptação de $A e$. scapularis em ambientes antropogênicos, onde tem demosntrado acentuada antropofilia, conquanto Forattini e col ${ }^{10}$. (1987) tenham destacado sua preferência por sangue de bovinos e eqüinos.

Os resultados aqui obtidos demonstram que Ae. scapularis tem densidades significativas das 18 às $19 \mathrm{~h}$, das 19 às $20 \mathrm{~h}$ e das 6 às $7 \mathrm{~h}$. Nos Estados de São Paulo 8,10 e Rio de Janeiro ${ }^{16}$ esse mosquito tem mostrado notável atividade no periodo noturno.

An. albitarsis também esteve presente em todos os horários de capturas, mostrando atividade significativa das 18 às $19 \mathrm{~h} \mathrm{em}$ isca humana. Esse inseto foi assinalado no rio Paraná ${ }^{3,4,12}$, Estado do Paraná, sempre em densidades elevadas, em isca humana, no crepúsculo da tarde. A importância de $A n$. albitarsis como vetor dos plasmódios da malária humana ${ }^{5}$ é secundária, sendo raras as localidades onde a transmissão se deve exclusivamente a essa espécie ${ }^{7}$. Todavia, a existência de $A n$. darling $i$ na região noroeste do Parana $a^{3}$, juntamente com $A n$. albitarsis, são fatos que devem ser considerados, pois nessa região tem ocorrido surtos epidêmicos de malária*. Além disso, há um movimento humano notável entre a citada região e Estados do norte do Brasil ${ }^{1,19,20}$, onde a malária é endêmica.

Ps. varipes esteve.presente de modo significativo das 18 às $20 \mathrm{~h}$ em isca humana. Por não

- Comunicação pessoal de G. Tomich - Superintendência das Campanhas de Saúde Pública (SUCAM) - Curitiba, PR. 
ter sido capturado nas armadilhas de Falcão, isso pode conferir-lhe um certo grau de antropofilia.

A quarta espécie mais freqüente em isca humana foi $C q$. lynchi, que prevaleceu entre $19 \mathrm{~h}$ e 20h. Poucos exemplares foram coletados nas armadilhas de Falcão. Assim, Ps. lynchi parece estar em situação similar a Ps. varipes quanto à antropofilia.

Ma. titillans (foi a quinta espécie mais freqüente em isca humana, com maior atividade preferentemente das 19 às $20 \mathrm{~h}$, tendo sido capturada em todas as armadilhas de Falcão. No Estado do Rio de Janeiro ${ }^{16}$, esse mosquito teve maior atividade no crepúsculo vespertino e foi assinalado em grande número, em isca humana e animal (Lourenço-de-Oliveira ${ }^{15}, 1984$ ). Posteriormente ${ }^{16,17}$, também no Rio de Janeiro, foi observado que Ma. titillans era uma das espécies mais freqüentes em isca humana. No Estado do Paraná ${ }^{4}$, no lago de Itaipu, esta espécie foi obtida em isca humana, no crepúsculo da tarde e em proporção similar à obtida no presente trabalho. Esses fatos sugerem que Ma. titillans é um mosquito que tem maior atividade no crepúsculo vespertino e hábito alimentar eclético.

Ad. squamipennis foi capturada quase exclusivamente nas armadilhas de Falcão, pois um único exemplar foi coletado em isca humana, o que constitui um forte indicativo de um possível caráter zoofilico e hábito noturno, dessa espécie, na região estudada.

Entre as espécies mais numerosas em isca humana, Ae. scapularis, além de ser dominante, foi a única que esteve presente em todas as capturas mensais, com densidade significativa no mês de abril. An. albitarsís, a segunda espécie de maior prevalência, só não foi capturada no mês de janeiro, tendo densidade significativa em julho. Ps. varipes e Cq. lynchi, terceira e quarta espécies predominantes em isca humana, compareceram irregularmente nas capturas mensais, com densidades significativas em setembro e fevereiro, respectivamente. A quinta espécie predominante, Ma. tittllans, não esteve presente em janeiro e maio, apresentando freqüencia significativa em setembro (Tabela 3).

Lourenço-de-Oliveira e col. ${ }^{17}$ (1985), no Estado do Rio de Janeiro, assinalaram que $A e$. scapularis e Ma.titillans compareceram em to- dos os meses, no decorrer de um ano de coletas. A primeira espécie teve maior densidade no mês de julho e a segunda nos meses de janeiro e novembro. Forattini e Gomes ${ }^{8}$ (1988) observaram a atividade de Ae. scapularis em todos os meses do ano, mas com maior incidência no mês de novembro.

De acordo com a análise dos resultados, em isca humana, é possível concluir que as espécies Ae. scapularis, An. albitarsis, Ps. varipes, Cq. lynchi e Ma. titillans foram coletadas: 1 . em número significativamente maior que as outras, com predomínio da primeira espécie; 2. em número significativo das 6 às 7 he das 18 às $20 \mathrm{~h}$, prevalecendo entre $18 \mathrm{e} 19 \mathrm{~h}$. Verificou-se ainda que houve diferença significativa do número de mosquitos de um mês para o outro.

Quanto aos culicídeos capturados com as armadilhas de Falcão verifica-se que: 1. houve diferença significativa no número de mosquitos coletados, comparando-se as quatro armadilhas; 2 . ocorreu diferença significativa entre as diversas espécies capturadas, sendo algumas bem mais abundantes; 3. Ad. squamipennis foi predominante, vindo em seguida $C x$. (Melanoconion) spp., An. albitarsis, $C x$. quinquefasciatus, Ae. scapularis, Ma. titillans e Cx. coronator.

\section{Agradecimentos}

À Jabur Agropecuária Ltda., proprietária da fazenda Sonho Real, e ao Sr. João de Lima, administrador dessa fazenda, pela ajuda imprescindivel à realização do presente trabalho.

TEODORO, U. et al. [Mosquitoes of peridomiciliary and extradomicillary environments in of southern Brazil]. Rev. Saúde Pública, 28: 107-15, 1994. Mosquitoes were collected on Sonho Real farm, Querência do Norte county, Paraná State, Brazil, using human bait and Falcão traps between June 1989 and May 1990. The fauna composition, monthly density, hours of major density, human attraction and presence of mosquitoes in domestic animal shelters were investigated. 5,923 mosquitoes of the genera Aedes, Aedomyia, Anopheles, Coquillettidea, Culex, Mansonia, Psorophora, Sabethes and Uranotaenia were collected. 33 species of mosquitoes were identified and among them Aedes scapularis, Anopheles albitarsis, Aedomyia squamipennis, Coquillettidea lynchi, Mansonia 
titillans e Coquilletlidea venezuelensis were predominant. All these species were captured mainly on human bait, except Aedomyia squamipennis that was captured in domestic animal shclters. With regard to all the mosquitoes captured $(5,923)$, their major period of activity was between 18 and 19 hours and $A$ pril was the month of grcatest density.

Keyrwords: Mosquitoes. Ecology, vectors.

\section{Referências Bibliográficas}

1. ALEGRE, M. \& MORO, D. A mobilidlade da população nas antigas áreas cafeciras do Norle do Paraná. Bol. Geog)., Maringá, (1): 28-73,1986.

2. BARBOSA, O. C.; 'THODORO, U.; LO\%OVEI, A.I.; LA SALVIA $\mathrm{F}^{\circ}$, V.; SPINOSA, R.P.; FERREIRA, M.E.M.C.; L.MA, E. M. de. Nota sobre culicideos adultos coletados na regiño sul do Brasil. Rev. Sañle Püblica, 27:214-6, 1993.

3. CONSOLIM, J. \& GAIVÃ̃ , J. '1". Sobre os anoliclinos do rio Paraníl. I.Densidade e regime do rio. Arq. Biol. Tecnol., 16:174-81, 1973.

4. CONSOLIM, J.; PELI.EGRINI, N.J.; LUZ, E. Culicídcos das áreas de Itaipu, Estado do l’araná, Brasil. I. Município de Foz do Iguaçu (Diptera - Culicidae). Acta Biol. Parana., Curitiba. (no prelo).

5. DEANE, L. M. Malaria vectors in Brazil. Mem. Inst. Oswaldo Cruz, 81(Suppl. 2): 5-11, 1986.

6. FALCÑO, A. R. Um novo modelo de armadilha luminosa de sucção para pequenos insctos. Mem. Inst. Oswaldo Cruz, 76:303-5,1981.

7. FERREIRA, E. Distribuiçĩo geográlica dos anofelinos no Brasil e a sua relaçĩo com o estado atual da crradicaçĩo da malária. Rev. Bras. Malariol, 16:32946,1964

8. FORATIINI, O. P. \& GOMES, A. dc C. Biting activity of Aedes scapularis (Rondoni) and Ilemagogus mosquitocs in Southern Brazil (Diptera - Culicidac). Rev. Saúle Pública, 22:84-93,1988.

9. FORATIINI, O. P.; GOMES, A. de C.; SANIOS, J.L.F.; GALATI, E.A.B.; RABELLO, E.X.; NATAL, D. Observações sobre a atividade de mosquitos Culicidae en mata residual no Vale do Ribcira, São Paulo, Brasil. Rev. Saliale Püblica, 15:557-86, 1981.

10. FORATTINI, O. P.; GOMLS, A. de C.; NATAI., D.; KAKITANI, I.; MARUCCI, D. Freqü̂̂ncia domiciliar e endofilia de mosquitos Culicidae no Vale do Ribeira, São Paulo, Brasil. Rev. Saúde Prública, 21:188-92, 1987.

11. FUNDAÇÃO IBGE. Geografia do Brasil: Região Sull. Rio de Janeiro, 1990. v. 2.

12. FUNDAÇÃO NACIONAL DE SAÚDE. Diretoria Regional do Paraná. Programa de controle sanitário da Usina Ilidrelétrica Taquaruçu; relatórios mensais de entomologia. Curitiba, 0.1/1991, 06/1991 e 08/1991. [mincografiado].

13. LOPES, J. Lcologia de mosquitos (Diptera - Culicidae) que procriam cm criadouros naturais e artificiais em área rural, Londrina e Cambé, Paraná, Brasil. Curitibat, 1992 ['Tese de Doutorado- Universidade Federal do Paraná].

14. LOPES, J.; BORSSA'TO, A. M.; PIRES, M. A. Entomofauna da mata Godoy. Culicidae (Diptera) procriando-se $\mathrm{cm}$ criadouros artificiais introduzidos na mata. Se mina, 8:67-9, 1987.

15. LOURENÇO-DE-OLIVEIRA, R. Alguns aspectos de ecoIogia dos mosquitos (Diptcra: Culicidae) de uma árci de planícic (Granjas Calábria), cm Jacarepaguá, Rio de Janciro. I. Freqüência comparativa das espécies en diferentes ambientes e métodos de coletas. Nem. Inst. Oswaldo Cruz, 79:179-90, 1984.

16. LOURIENS.O-DI:-OLIVLIRA, R. \& SILVA, T.F. Alguns as pectos da ecologia dos mosquitos (Diptem: Culici(ac) de uma átea de planície (Granjas Calábria), em Jacarepaguá, Rio de Janciro. III. Preferência horária das lêmeas para o hematolagismo. Mem. Inst. Osuaklo Cinz, 80:195-201,1985.

17. L.OURENÇO-DE-OLIVEIRA, R.; SILVA, T. F.; IIEYDEN, R. Alguns aspectos da ccologia dos mosquitos (Diptcıa: Culicidac) de uma área de planície (Granjas Calábria), cm Jacarepaguá, Rio de Janciro. II. Freqüência mensal e no ciclo lunar. Mem. Inst. Oswaldo Critz, 80:123-33,1985.

18. MACK, R. Geografia fisica do Estado do Paraná. 2" ed. Rio de Janciro, José Olympio Ed./ Secr. Cult. Esp. Est. Paraná, 1968.

19. MARQUES, A. C. Migration and dissemination of malaria in Brazil. Mem. Inst. Oswatlo Crttz, 81 (Suppl. 2) :17-30,1986.

20. MARQUeS, A. C.; PINIIEIRO, E. A.; SOUZA, A. G. de. Un estudo sobre a dispersão de caso de malária no Brasil. Rev. Bras. Malariol., 38:51-75,1986.

21. RACIIOU, R. \& RICCIARDI, I. I. Contribuição ao conhecimento da distribuição geográfica dos anofelinos no Brasil: Isstado do Paranáa (distribuição por municípios c localidades). Rev. Bras. Mfalariol, 3:42348,1951 .

22. UNIVERSIDADE ESTADUAL DE MARINGÁ. Degradação ambiental no norocste do Paraná. Bol. Geogr, Maringá, 9:1-57,1991.

Recebido para publicação em 15.7.1993

Reapresentado em 2.12.1993

Aprovado para publicação em 5.1.1994 